\title{
Long Term Survivor of Vaginal Primary Malignant Melanoma \\ Kalam $\mathrm{F}^{1}$, Pervin $\mathrm{S}^{2}$
}

DOI: https://doi.org/10.3329/jafmc.v15i1.48660

\begin{abstract}
Primary malignant melanoma of the vagina is a rare entity which constitutes less than $3 \%$ of vaginal cancer and only $0.3-0.8 \%$ of all melanomas in women. It has a high risk of local recurrence and distant metastasis. Once a recurrence is noted, survival is extremely poor. This article reports a case of primary vaginal melanoma with longer survival. As long-time survival of stage II vaginal melanoma is a rare phenomenon, this case is a unique one in Bangladesh; emphasizing on both importance of routine gynaecological examination for any suspicious pigmented lesions in the vagina as well as the necessity of further research on validating treatment protocols.
\end{abstract}

Key-words: Primary vaginal malignant melanoma, Malignant melanoma, Chemotherapy.

\section{Introduction}

Malignant melanoma of the vagina is a rare, aggressive malignancy of poor prognosis that affects women in their 6th and 7th decade of life ${ }^{1,2}$. Melanomas are tumours that arise from melanocytes or pigment cell. A common form of melanoma is primary mucosal melanoma that is found in the mucosal lining of the urogenital tract, respiratory tract and genitourinary tract. Vaginal primary malignant melanoma (VPMM) is one such type of mucosal malignant melanoma that is very rare. It is thought to arise from aberrantly located melanocytes of the vaginal epidermis and accounts for $0.3-0.8 \%$ of all malignant melanomas, $2-5 \%$ of female genital tract melanomas and less than $3 \%$ of all vaginal malignancies ${ }^{3,4}$.

\section{Case Report}

A 46 years old female, para-4 attended to Gynaecologic oncology unit of Institute of Cancer Research and Hospital (NICRH) on 3.1.15 as a known case of vaginal melanoma. She first reported at Mymensingh Medical College (February 2014) with the complaints of per vaginal discharge with itching and black pigmented nodule was found during the $\mathrm{p} / \mathrm{v}$ examination. Wide local excision of the vaginal nodule was done on 18.05 .14 and tissues were sent for histopathology. Her report revealed Malignant Melanoma. There she received 4 cycles adjuvant chemotherapy with Cisplatin and Dacarbazine (DTIC) (13.12.14) and was referred to NIRCH.

At $\mathrm{NIRCH}$, the patient was reviewed again and $\mathrm{p} / \mathrm{v}$ examination showed black pigmentation with a raised irregular surface on both anterior and posterior vaginal wall, small growth over the cervical lip. Then decision of surgery was taken for vaginal melanoma stage-II. On 17.1.15 Radical Hysterectomy with Bilateral Pelvic Lymphadenectomy with Total Vaginectomy with
Inguinal lymphadenectomy was performed. Histopathology report confirmed Malignant melanoma of vaginal wall, resection margin was free of tumour, uterine cervix showed metastatic foci of the malignant melanoma, adenomyosis in the myometrium, sectioned made from the internal os and vaginal tissue attached with the uterus were free of tumour, both sided ovaries and fallopian tubes were unremarkable. Parametrium was free of tumour, all pelvic lymph nodes and inguinal lymph nodes showed reactive changes. Then the patient received 25\# External Beam Radiation Therapy (EBRT) according to the decision of the tumor board. She was under regular follow up with disease-free survival.

About 8 months later, on 27.8.16 during follow up period again black pigmentation in the area of the posterior vaginal wall was noticed and wide local excision was done on 3.10.16. The histopathology report revealed squamous hyperplasia. The follow up continued and the patient remained asymptomatic. On 14.7.18 multiple black pigmented deposit $(1.5 \times 1.5) \mathrm{cm}$ with smooth surface were noticed in the area of posterior margin of the vault and with the base of the bladder. Surgical excision was done on 19.9.18. Histopathology report confirmed: Squamous papilloma. The present case study describes the clinical scenario and surveillance of this patient with primary malignant melanoma of the vagina. Now she is alive and without evidence of VPMM sixty-one months after the occurrence; though benign lesion of vaginal wall occurs during this period.

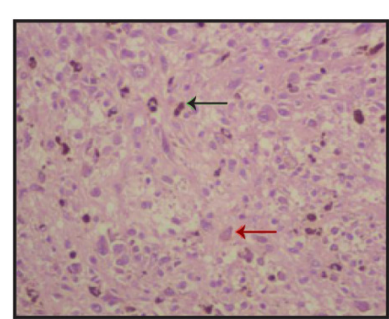

(a)

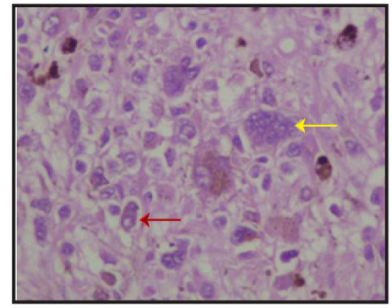

(c)

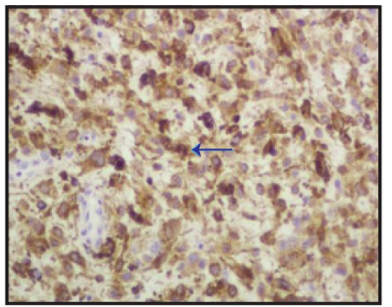

(b)

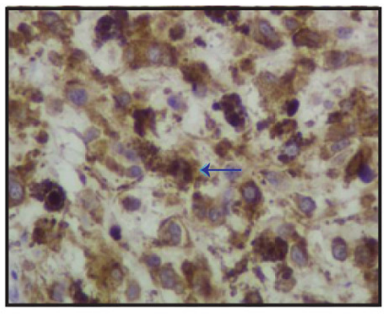

(d)
Figure-1: Predominant cell type was epithelioid (red arrow), multinuclear giant cell (yellow) abundant melanin deposit (green), the neoplastic cells expressed Tyrosinase (blue)

1. Dr Farhana Kalam, MBBS, Junior Consultant of Obstetrics and Gynaecology, National Institute of Cancer Research and Hospital (NICRH), Mohakhali, Dhaka (E-mail: ovikalam@yahoo.com) 2. Dr Shahana Pervin, MBBS, Associate Professor of Gynaeoncology, NICRH, Mohakhali, Dhaka. 


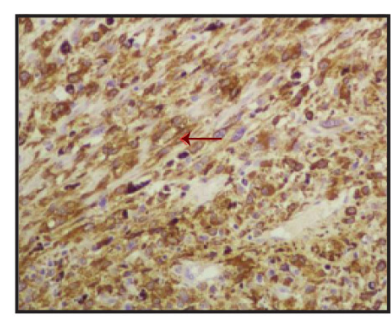

(a)

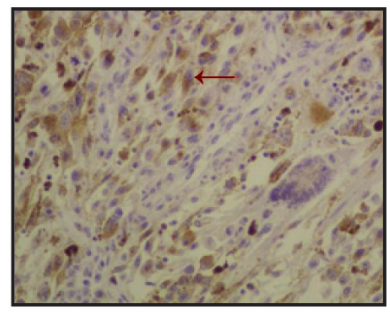

(c)

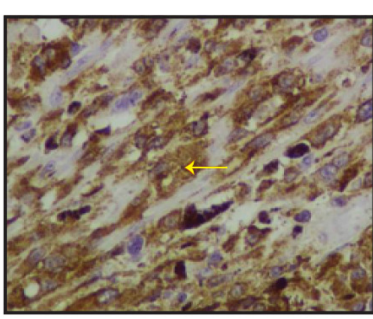

(b)

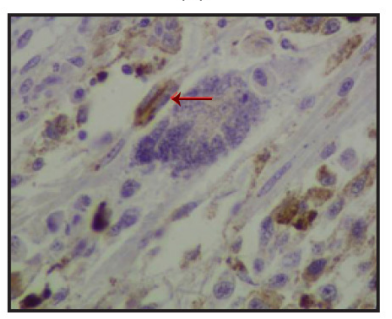

(d)
Figure-2: Immunophenotype of tumour cells. The neoplastic cells expressed melanin $\mathrm{A}(\mathrm{a}, \mathrm{b})$ and $\mathrm{s}-100(\mathrm{c}, \mathrm{d})$

\section{Discussion}

It is a rare gynaecological malignancy that is associated with potential risk of recurrence, distant metastases and short survivability. Melanoma of the vagina may be misdiagnosed sometimes by gynaecologist due to the rarity of the case and lack of awareness of the potential diagnosis. Immunohistochemical staining positive for vimentin, protein S-100, Melan A, HMB-45 is used to confirm the diagnosis ${ }^{5-7}$. In this case, the pathological results indicated a malignant melanoma and immunohistochemical analyses demonstrated S-100, Melan A positive staining which further confirmed the diagnosis. Conservative wide local excision, radical surgery, radiotherapy, immunotherapy and chemotherapy are the currently proposed therapeutic options, which can be used in combination or individually for vaginal primary melanoma ${ }^{2,7}$. Other potential prognostic factors including age, FIGO stage, the location of the tumour, invasion of depth, pigmentation, ulceration, histology, cell type, number of mitoses, type of surgical procedure, adjuvant radiotherapy, chemotherapy ${ }^{5,6}$.

Conversely, Petru et al found that histological features, such as cell type, mitotic count, ulceration, vessel and lymphatic involvement didn't correlate with survival time. The treatment should aim to complete resection of the tumour and evaluation of the related lymph nodes for tumour involvement ${ }^{6}$. The surgical approaches including wide local excision, total or radical vaginectomy with en block removal of the involved pelvic organs has been considered the most effective curative options, which could increase the chances of a longer survival than that treated non-surgically. Despite these treatment options, the recent SEER (Survailance, Epidemiology, End-Result program) study reported an overall survival at 2 years and 5 years are of $24 \%$ and $15 \%$ respectively ${ }^{4}$. This patient is very much fortunate that she remains disease free for sixty-one months. The primary treatment modality is important in the prognosis of the disease. Overall radiotherapy may be used as a rescue treatment to improve the prognosis of patients with vaginal melanomas ${ }^{6}$. Currently, there have been only a few reports on the efficacy of chemotherapy though it's effect as a primary or adjuvant therapy on patient survival has not been yet determined. However, Dacarbazine (DTIC) has been the standard of care for many years in patients with response rates ${ }^{2}$ of $7.5 \%$ to $12.1 \%$.

\section{Conclusion}

In low resource setting countries like Bangladesh where maximum patients come from lower socio-economic group, it is a great challenge to provide optimum treatment. Even though optimal treatment against a very rare and aggressive disease is possible by doctor's vigilance, patient's compliance, thorough and regular follows up. The case of this cancer survivor is a bright example of it. So, attention should be given to any suspicious pigmented lesions in the vagina in routine gynaecological examination. Further research is needed to identify the aetiology and improve the outcome of this aggressive disease.

\section{References}

1. Creasman WT, Phillips JL, Menck HR. The National Cancer Data Base report on cancer of the vagina. Cancer 1998; 83(5):1033-40.

2. Piura B. Management of primary melanoma of the female urogenital tract. Lancet Oncol 2008; 9(10):973-81.

3. Weinstock MA. Malignant melanoma of the vulva and vagina in the United States: Patterns of incidence and population-based estimates of survival. Am J Obstet Gynecol 1994; 171(5):1225-30.

4. Hacker NF, Eifel PJ. Berek \& Hacker'sGynecologic Oncology, 6th ed, Philadelphia 2015:617-618.

5. Chen $\mathrm{L}$, Xiong $\mathrm{Y}$, Wang $\mathrm{H}$ et al. Malignant melanoma of the vagina: A case report and review of the literature. Oncology Letters 2014; 8(4):1585-8.

6. Petru E, Nagele F, Czerwenka K et al. Primary malignant melanoma of the vagina: Long-term remission following radiation therapy. Gynecol Oncol 1998; 70(1):23-6.

7. Cardenes HR, Jeanne M. Principles and Practice of Gynecologic Oncology, 6th ed, Wolters kluwer, Philadelphia 2015:584-5, 566-7. 